\title{
Coexistence of Spheres and Rods in Micellar Solution of Dodecyldimethylamine Oxide
}

\author{
Pinaki R. Majhi, ${ }^{\dagger}$ Paul L. Dubin, ${ }^{*}$ Xianhua Feng, ${ }^{\ddagger}$ and Xuhong Guo ${ }^{\S}$ \\ Department of Chemistry, Indiana University-Purdue University Indianapolis, 402 North Blackford Street, \\ Indianapolis, Indiana 46202
}

\begin{abstract}
F. A. M. Leermakers"
Laboratory of Physical Chemistry and Colloid Science, Wageningen University, Dreijenplein 6, 6703 HB Wageningen, The Netherlands
\end{abstract}

\author{
Christophe Tribet $^{\perp}$ \\ Laboratoire de Physico-Chimie Macromoleculaire, Universite Paris 6, UMR 7615, ESPCI, 10 rue Vauquelin, \\ F-75231 Paris Cedex 05, France
}

Received: November 11, 2003; In Final Form: March 10, 2004

\begin{abstract}
Micelles of dimethyldodecylamine oxide (DMDAO) are known to exhibit sphere-to-rod transitions as a function of $\mathrm{pH}$ and ionic strength. Long micelles are stabilized at $\mathrm{pH}$ corresponding to half-protonation, because hydrogen bonding between nonionic and protonated monomers yields an effectively double-tailed monomer whose geometry favors cylindrical growth. Dynamic light scattering (DLS) was used to follow particle size distribution as a function of $\mathrm{pH}$, ionic strength $(I)$, and surfactant concentration. The key finding was the coexistence of spherical micelles with rodlike ones at $4<\mathrm{pH}<6$ and $I>0.2 \mathrm{M}$. These observations have been verified by varying the algorithm used for the Laplace transformation of the autocorrelation function and also with different DLS systems. The effect of surfactant concentration was used to confirm the absence of any influence of micelle-micelle interaction on the dynamics of diffusion. A molecular level self-consistent field analysis of finite size rodlike micelles confirms the idea that the endcaps are swollen with respect to the cylindrical part. The theoretical results support the coexistence of rods and spherical micelles, i.e., the existence of gaps in the size distribution of wormlike micelles. The cause of coexistence has been explained in terms of the instability of dumbbell-like micelles with domains of negative curvature (neck). The endcap energy is shown to be given (in first order) by the grand potential of spherical micelles that coexist with the wormlike micelles.
\end{abstract}

\section{Introduction}

The dependence of the size of mixed micelles on the overall surfactant composition is an important and interesting topic in the field of surfactant chemistry, both with regard to theory and also in view of the practical applications of mixed surfactant systems. ${ }^{1}$ Indeed, pure surfactants are rarely encountered in industrial applications, due to both economic factors and "synergistic" effects. While most such systems involve chemically different surfactant monomers, typically mixtures of ionic and nonionic ones, dimethyldodecylamine oxide (DMDAO) is unique in that this single surfactant forms micelles comprised of mixtures of protonated (cationic) and deprotonated (nonionic) monomers depending on the $\mathrm{pH}$. Such $\mathrm{pH}$ adjustment also provides a way for micelle surface charge density to be varied precisely through changing the degree of protonation $(\beta)$ of the amine oxide headgroup. Because of the concomitant electrostatic

\footnotetext{
* Corresponding author. E-mail: dubin@ @ chem.iupui.edu.
† Present address: Department of Pharmaceutical Chemistry, School of Pharmacy, University of California, San Francisco, 513 Parnassus Ave., Box 0446, San Francisco, CA 94143. E-mail: pinakirm@yahoo.com.

† Present address: Department of Chemical Engineering, McMaster University, Hamilton, ON L8S 4L7, Canada.

$\S$ Present address: Department of Chemical Engineering, Princeton University, Princeton, NJ.

"E-mail: frans.Leermakers@wur.nl.

${ }^{\perp}$ E-mail: Christophe.Tribet@espci.fr.
}

and hydrogen-bonding interactions, DMDAO micelles adopt spherical or cylindrical geometry, depending on $\beta$ and ionic strength. ${ }^{2,3}$ As a result of this complex and unique behavior, the dependence of the size and shape of DMDAO micelles on $\mathrm{pH}$ and ionic strength has been studied extensively for several decades. $^{2-28}$

DMDAO micelles show characteristic maxima or minima in several physical properties at or near the point of half-ionization $(\beta=0.5)$ : (1) The micelle size goes through a maximum at half-ionization, and the aggregation number or the micelle molecular weight exhibits corresponding maxima; $;^{2,4,22,24}$ similarly, the viscosity attains a maximum ${ }^{14}$ near $\beta=0.5$. (2) A minimum in the critical micelle concentration has also been observed at half-ionization. ${ }^{24,28}$ (3) At this same point, the surface tension (at the $\mathrm{cmc}$ ) and the surface excess at the airwater interface show a minimum and maximum, respectively. ${ }^{23,28}$ (4) Last, infrared spectra $^{18}$ at $\beta=0.5$ show a minimum in the frequency of the $\mathrm{CH}$ stretching band, and disappearance of the absorption band at approximately 1330 $\mathrm{cm}^{-1}$, assigned to the combination of $\mathrm{CNO}$ bending and $\mathrm{CH}_{2}$. These widely varied observations have been explained on the basis of hydrogen-bond formation between the protonated and deprotonated species of DMDAO which attains a maximum when the number of protonated and unprotonated species are equal. $3,13,24$ The strong interaction between the amine oxide 


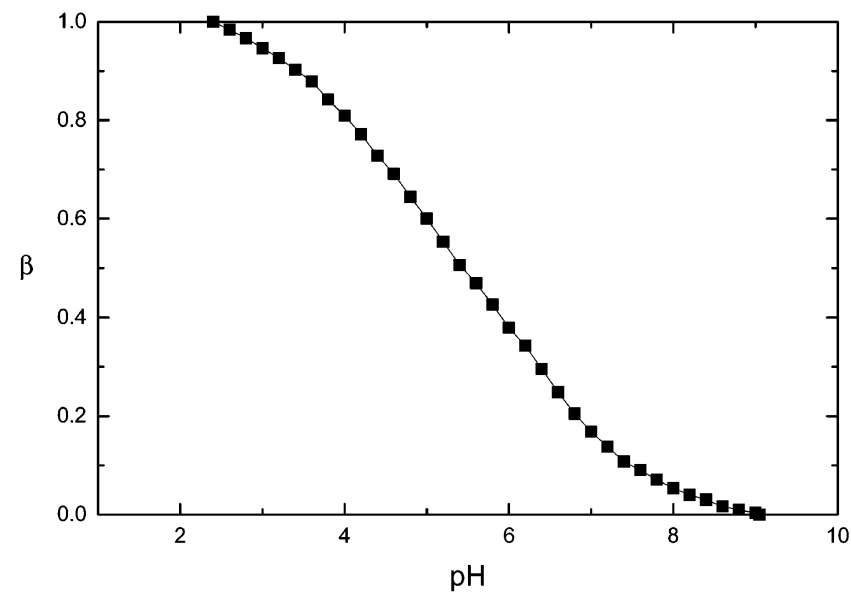

Figure 1. Titration of $25 \mathrm{mM}$ DMDAO in $0.80 \mathrm{M} \mathrm{NaCl}$ solution with $0.80 \mathrm{~N} \mathrm{HCl}$.

acceptor group of the deprotonated monomer and the $\mathrm{N}$ hydroxyammonium donor group of the protonated species leads to the formation of hydrogen-bonded dimers with a reduction in the effective headgroup per micellized surfactant molecule. In a manner similar to double-tailed surfactants, these species accommodate surfaces of low curvature, and consequently stabilize cylindrical micelles.,21-22,29 Consequently, one observes, as $\beta=0.5$ is approached from either direction, a transition to extended rodlike micelles. , $^{3,28}$

It is generally assumed that micelles may grow monotonically from spheres to rods with change of various parameters, ${ }^{30}$ but a few studies suggest otherwise. Cryo-TEM and pulse gradient spin-echo NMR methods revealed the coexistence of spherical and threadlike micelles in dilute solutions of the nonionic surfactant octadecylamide oligo(oxyethylene ether). ${ }^{31}$ Similar coexistence of spheroidal and long rodlike micelles was seen by cryo-TEM with a dimeric (Gemini) surfactant within a definite concentration range. ${ }^{32}$ Imae et al. observed two modes in the electrophoretic light scattering spectra of amine oxide micelles, ${ }^{21}$ but did not clearly account for the zero mobility value of one of them. In our present study, we report on the coexistence of spherical and rodlike micelles in the DMDAO system observed by dynamic light scattering, along with a hypothesis for this coexistence and a detailed theoretical treatment.

\section{Experimental Section}

Materials. $N, N$-Dimethyldodecylamine- $N$-oxide (DMDAO) was from Fluka (Product \# 40234) purity $>98 \%$. Solid $\mathrm{NaCl}$ and standard $\mathrm{NaOH}$ and $\mathrm{HCl}$ solutions were from Fisher Scientific (Pittsburgh, PA). Mill-Q water was used throughout the work.

Potentiometric Titrations. pH titrations of 25 or $50 \mathrm{mM}$ DMDAO were made by addition of standardized $\mathrm{HCl}$ or $\mathrm{NaOH}$ with Gilmont microburets using a Beckman $\varnothing 34 \mathrm{pH}$ meter equipped with a Beckman combination electrode, at $25 \pm 1$ ${ }^{\circ} \mathrm{C}$, under $\mathrm{N}_{2}$ and magnetic stirring, using procedures described elsewhere. ${ }^{3}$ Appropriate blank volumes were subtracted to correct for the hydrolysis of DMDAO (at high $\mathrm{pH}$ ) or the dissociation of $\mathrm{DMDAOH}^{+} \mathrm{Cl}^{-}$(at low $\mathrm{pH}$ ). Both high- and low-pH end points were detected as maxima in $\Delta \mathrm{pH} / \Delta \mathrm{mL}$, corresponding to $\beta=0$ and $\beta=1$, respectively, where $\beta$ is the degree of protonation. The $\mathrm{pH}$ values are converted to the degree of protonation $\beta$ using the $\mathrm{pH}$ titration curve, one example being shown in Figure 1.
Dynamic Light Scattering (DLS). Solutions of DMDAO, 5-100 mM, ionic strengths from 0.2 to $0.8 \mathrm{M}$, and $3<\mathrm{pH}<$ 9 were prepared and filtered with $0.22 \mu \mathrm{m}$ filters (Sartorius AG, Germany) before measurements. DLS was carried out with (1) a Brookhaven instrument (Holtsville, NY) equipped with a BIC9000AT digital autocorrelator and a $488 \mathrm{~nm} 100 \mathrm{~mW}$ argonion laser; (2) a DynaPro-801 system (Protein Solutions Inc., Charlottesville, VA) equipped with a $30 \mathrm{~mW}$ solid-state 780 $\mathrm{nm}$ laser and an avalanche photodiode detector; (3) an ALV instrument ${ }^{33}$ comprised of a Malvern goniometer, and ALV500 multibit and multitaux digital autocorrelator, ALV800 photomultiplier, and a $514.5 \mathrm{~nm} 3 \mathrm{~W}$ argon-ion laser (SP2020, Spectra Physics, CA). The later system was used in very limited study to verify observations of bimodality from the other two instruments. Measurements were usually carried out at $25 \pm 1$ ${ }^{\circ} \mathrm{C}$ and a $90^{\circ}$ scattering angle, with additional angle dependence studied using system (1).

In the self-beating mode of dynamic light scattering, the measured time correlation function, $G^{(2)}(\tau, q)$, of a collection of monodisperse particles or molecules undergoing Brownian motion shows a Gaussian-type distribution and it has the form

$$
G^{(2)}(\tau, q)=A\left(1+b\left|g^{(1)}(\tau, q)\right|^{2}\right)
$$

where $g^{(1)}(\tau, q)$ is the first-ordered scattered electric field time correlation function; $\tau$, the delay time; $A$, the experimental baseline; $b$, a spatial coherence factor which depends on the number of coherence areas generates the signal $(0<b<1)$; and $q$, the amplitude of the scattering vector, given by $q=(4 \pi n /$ $\lambda) \sin (\theta / 2)$, with $n$ and $\theta$ being the refractive index of the scattering medium and the scattering angle, respectively.

The electric field correlation function depends on the Fourier transform of the fluctuating number of the particles or molecules. For the center of mass diffusion of identical particles, the following single relationship holds:

$$
\left|g^{(1)}(\tau, q)\right|=\exp (-\Gamma \tau)
$$

where $\Gamma$ is the decay or relaxation rate and $D$ is the translational diffusion coefficient. The term $\tau_{\mathrm{r}}=1 / \Gamma$ is known as the decay or relaxation time.

For a polydisperse system, the correlation function $g^{(1)}(\tau, q)$ can be expressed as the integral of the exponential decays weighted over the distribution of the relaxation times:

$$
\left|g^{(1)}(\tau, q)\right|=\int_{0}^{\infty} G(\Gamma, q) \exp (-\Gamma(q) \tau) \mathrm{d} \Gamma
$$

where $G(\Gamma, q)$ is the normalized distribution of line width $\Gamma$ measured at a fixed value of $q$. The problem is then to find out $G(\Gamma, q)$. Several numerical methods have been developed to calculate $G(\Gamma, q)$. In the present study, the autocorrelation functions obtained from the Brookhaven, Protein Solutions, and ALV systems were analyzed using NNLS $^{34}$ or Cumulants, ${ }^{35}$ CONTIN $^{36}$ and REPES ${ }^{37}$ fitting procedures, respectively, to calculate $G(\Gamma, q)$. A discussion of the comparative features of these different algorithms may also be found in ref 38 .

From eq 3 , the mean relaxation time, $\left\langle\tau_{\mathrm{r}}\right\rangle$, defined as the area of $\left|g^{(1)}(\tau, q)\right|$, is given by

$$
\left\langle\tau_{\mathrm{r}}\right\rangle=\left(\int_{0}^{\infty} \Gamma G(\Gamma, q) \mathrm{d} \Gamma\right) /\left(\int_{0}^{\infty} G(\Gamma, q) \mathrm{d} \Gamma\right)
$$

This $\left\langle\tau_{\mathrm{r}}\right\rangle$ value can be resolved from each of the distribution modes of $G(\Gamma, q)$ as the first moment of the normalized relaxation spectrum. Therefore, the apparent translational diffusion coefficient, which corresponds to each value of $\left\langle\tau_{\mathrm{r}}\right\rangle$, can 


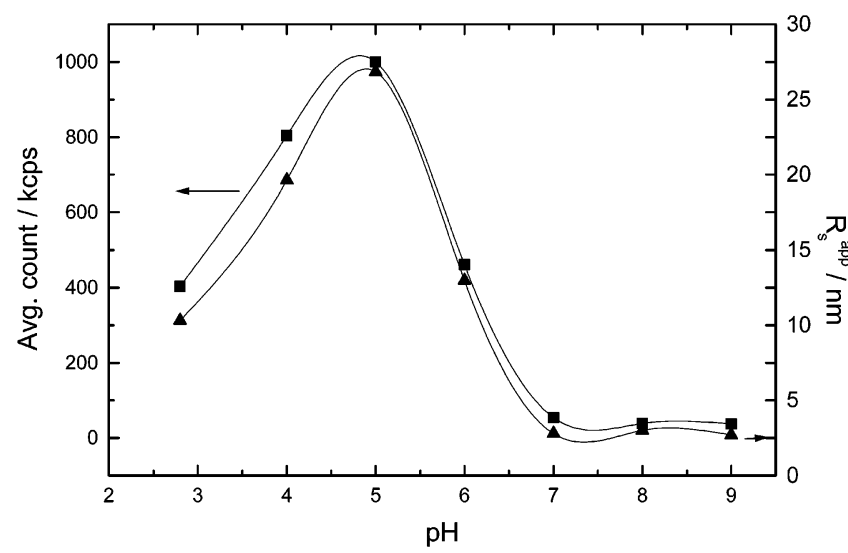

Figure 2. Scattering intensity and average radius $\mathrm{vs} \mathrm{pH}$ for $25 \mathrm{mM}$ DMDAO at $I=0.8 \mathrm{M}$ (Brookhaven system).

be calculated using the relation $D=\Gamma / q^{2}=1 /\left(q^{2} \tau\right)$. The apparent hydrodynamic radius $\left(R_{\mathrm{S}}\right.$ app $)$ can then be estimated via the Stokes-Einstein equation

$$
R_{\mathrm{s}}^{\text {app }}=k T / 6 \pi \eta D
$$

where $k$ is the Boltzmann constant, $T$ is the absolute temperature, and $\eta$ is the solvent viscosity.

\section{Results and Discussion}

Figure 2 shows the pH-dependence of the apparent Stokes radius $R_{\mathrm{S}}$ app from Cumulants analysis of the autocorrelation function, along with the total scattering intensity, for $25 \mathrm{mM}$ DMDAO at $I=0.80 \mathrm{M}$. Both results confirm numerous observations of a maximum in micelle size near half-ionization. ${ }^{2,4,24}$ The value of $R_{\mathrm{s}}$ app $=3 \mathrm{~nm}$ at high $\mathrm{pH}$ is consistent with the formation of small nonionic spherical micelles, as reported elsewhere, ${ }^{3}$ and the high curvature is likely due to dipole-dipole repulsions among the deprotonated amine oxide headgroups. The larger apparent size at low $\mathrm{pH}$ probably corresponds to short ellipsoidal or capsule-shaped cationic micelles. ${ }^{3,28}$ Upon increase of $\mathrm{pH}$, these become rodlike. ${ }^{3}$

A more detailed examination of the results is provided in Figure 3, which displays apparent particle size distributions, obtained via NNLS analysis of autocorrelation functions. While the results at high and low $\mathrm{pH}$ are in agreement with the cumulants data of Figure 2, the bimodal distributions between pH 4 and 6 are evident, suggesting the presence of two different particle sizes, namely spherical and rodlike micelles. However, this interpretation rests on some assumptions about the significance of the QELS data. The first is that the QELS signal arises from true translational diffusion, and this is borne out by the angular dependence of the average relaxation rate (reciprocal of the Cumulants-average diffusion time constant) shown in Figure 4. The linearity of $\Gamma$ vs $q^{2}$ (where $q$ is the magnitude of the scattering wave vector), and the agreement of particle radii extracted from the slopes at $\mathrm{pH}>6$ with the value of $R_{\mathrm{s}}{ }^{\text {app }}=$ $3 \mathrm{~nm}$ obtained from the $90^{\circ}$ cumulants measurement, both support the interpretation of decay constants in terms of translational diffusion coefficients.

While the interpretation of the data at high and low $\mathrm{pH}$ is straightforward and in good agreement with micelle aggregation numbers from other sources, ${ }^{22,24}$ interpretation of the fast decay mode at $4<\mathrm{pH}<6$ in terms of a spherical micelle must be considered vis-à-vis the possibility of a fast decay corresponding to network relaxations in systems of entangled rodlike micelles. ${ }^{27}$ In this case, an increase in micelle concentration should lead to

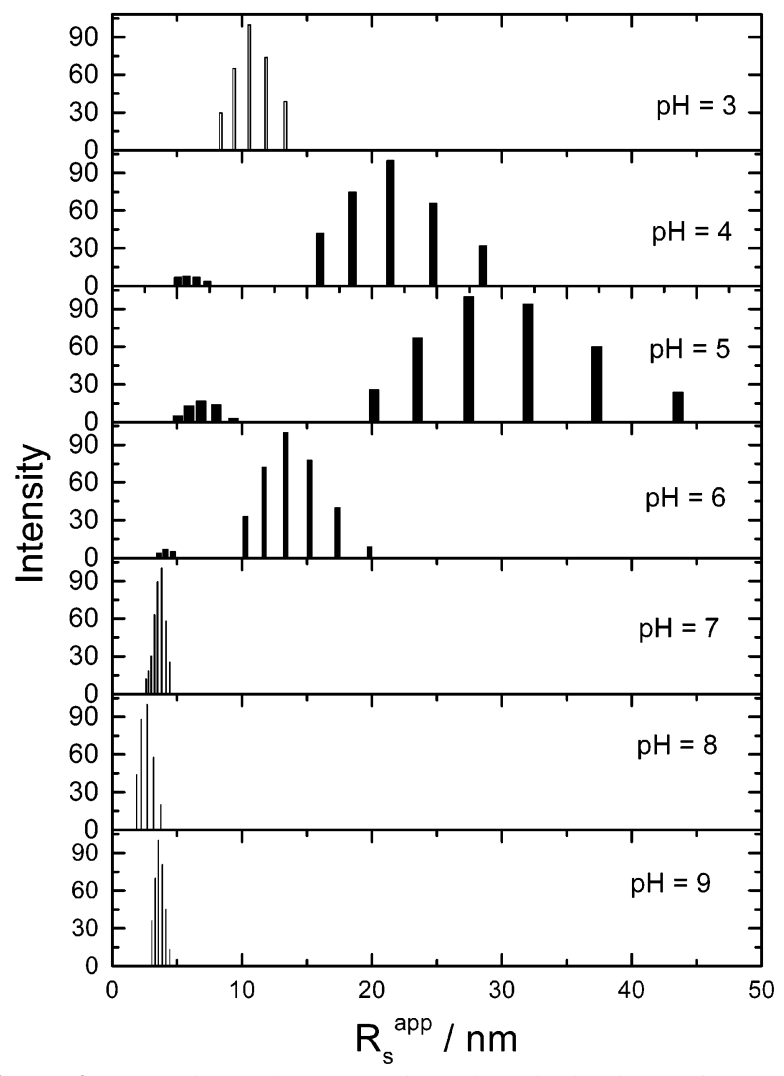

Figure 3. Intensity weighted particle size distributions of $25 \mathrm{mM}$ DMDAO $(I=0.8 \mathrm{M})$ at different $\mathrm{pH}$ (Brookhaven system).

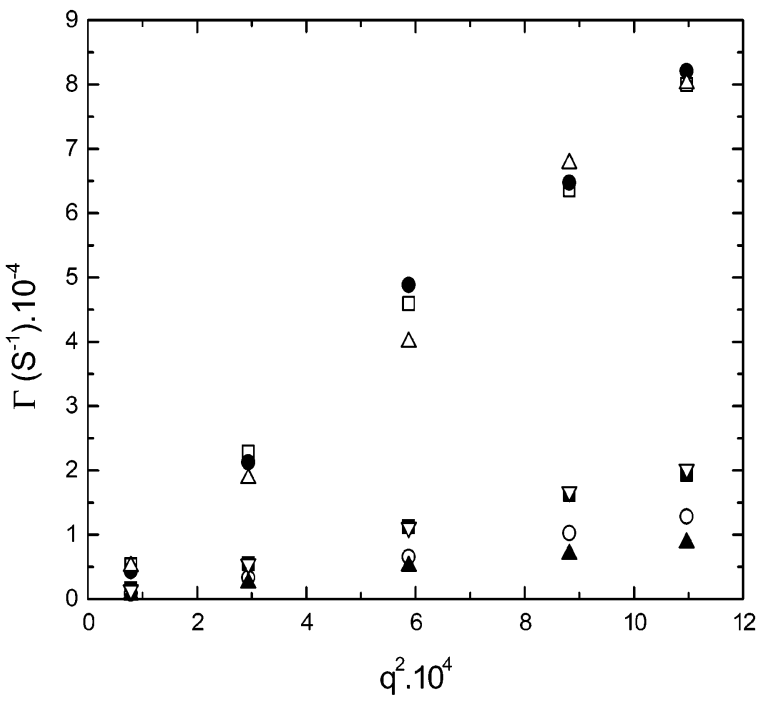

Figure 4. Angular dependences of the relaxation rate $\Gamma$ for $25 \mathrm{mM}$ aqueous DMDAO solutions with ionic strength $0.8 \mathrm{M}$ at pH $3(\mathbb{\square}), 4$ $(\bigcirc), 5(\mathbf{\Delta}), 6(\nabla), 7(\square), 8(\bullet), 9(\Delta)$; (Brookhaven system).

a decrease in network mesh size and a concomitant increase in the fast decay diffusion coefficient, so that $R_{\mathrm{S}}$ app would decrease with increasing concentration. As seen in Figure 5, the diffusion coefficient of the fast decay in fact decreases slightly with concentration: the value of $R_{\mathrm{S}}$ app increases from 5 to $7 \mathrm{~nm}$ as the concentration increases from 5 to $25 \mathrm{mM}$. We can furthermore estimate the overlap concentration $c^{*}$ using the aggregation number for DMDAO of 400 reported $^{22}$ at $I=0.2$ $\mathrm{M}, \mathrm{pH}=5$, and the relation

$$
c^{*}=3 M /\left(4 \pi N_{\mathrm{av}} R_{\mathrm{g}}{ }^{3}\right) \approx 3 M /\left[4 \pi N_{\mathrm{av}}\left(1.5 R_{\mathrm{h}}\right)^{3}\right]
$$

For rodlike micelles with $R_{\mathrm{h}}=25 \mathrm{~nm}$, we calculate $c^{*}=25$ 


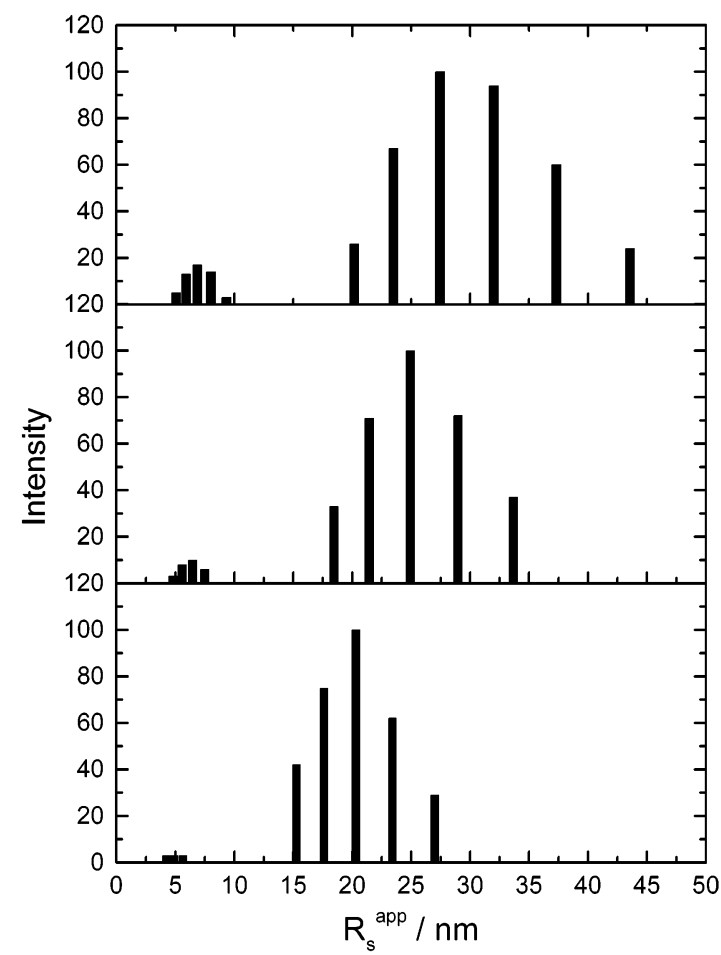

Figure 5. Intensity weighted particle size distributions of DMDAO at $\mathrm{pH} 5$ and $I=0.8 \mathrm{M}$ at concentrations 25,10 , and $5 \mathrm{mM}$ (from top to bottom); (Brookhaven system).

$\mathrm{mM}$. This, however, is in excess of most of the concentrations employed for Figure 5 so as to render overlap unlikely. Finally, none of the DMDAO solutions studied in this work, with concentrations up to $100 \mathrm{mM}$, display a significant increase in viscosity. Taken together, these observations support the interpretation of the bimodal distributions at $4<\mathrm{pH}<6$ as coexistence of short and long micelles.

Since the deconvolution of autocorrelation functions can be delicate, the results could be put on firmer ground by demonstrating the robustness of the apparent size distributions. Results were therefore generated on instruments other than the BIC9000AT and using algorithms different from NNLS. DMDAO solutions with concentrations ranging from 5 to $100 \mathrm{mM}$, and ionic strengths from 0.2 to $0.8 \mathrm{M}$ were analyzed with a Protein Solutions Model 801 instrument using CONTIN. Results are shown in Figure 6, where the $\mathrm{pH}$ values are replaced by degree of protonation $\beta$, and effects of concentration and ionic strength on the bimodality are additionally considered. Here for clarity and to accommodate all data in a single plot, each distribution mode (as shown in Figures 3 and 5) was replaced by a single $R$ value, obtained from the average value of $\Gamma$ for that mode. The presence, at any given ionic strength and surfactant concentration, of two $\mathrm{R}_{\mathrm{s}}$ app values for a single $\beta$, representing a bimodal distribution, was observed at $0.3<\beta<0.8$ for I $>$ 0.2M. Additional experiments were carried out with the ALV instrument. Analysis of the data with the software REPES also confirmed the bimodal distributions obtained by NNLS and CONTIN, and the three sets of data for $25 \mathrm{mM}$ DMDAO in $0.8 \mathrm{M} \mathrm{NaCl}$ are compared in Table 1 . Despite some inconsistencies, the results all support the presence of spherical/ellipsoidal micelles with $\mathrm{R}_{\mathrm{s}}$ app ranging from 3 to $7 \mathrm{~nm}$, coexisting in the $\mathrm{pH}$ range 4-6 with cylindrical micelles of 15 to $40 \mathrm{~nm}$.

The bimodal micelle size distributions seen here indicate destabilization of short cylindrical micelles with hydrodynamic radii on the order of $10 \mathrm{~nm}$. Relatively few reports of such nonmonotonic distributions have appeared. Khan et al. ${ }^{31}$ used
NMR self-diffusion and cryo-TEM to observe small (2-3 nm) spherical micelles of the nonionic surfactant octadeylamide oligo(oxyethylene ether), with dimensions independent of concentration, coexisting with long $(12-60 \mathrm{~nm})$ micelles whose size increased with concentration. Talmon and co-workers ${ }^{32}$ studied the dimeric (gemini) surfactant 12-2-12 (dimethylene 1,2-bis(dodecyl dimethylammonium bromide)) by Cryo-TEM and observed coexistence of spherical and long threadlike micelles. Elongated micelles of intermediate dimensions were rare, with a near absence of micelles 2-3 times larger than the spherical micelles (in the present case corresponding to micelles with radii of about $10 \mathrm{~nm}$ ). Talmon et al. also observed radii of the endcaps of the cylindrical micelles larger than those of the cylindrical regimes.

The DMDAO system, in contrast to the systems mentioned above and described in refs 31-32, allows for disproportionation as a means to further stabilize mixtures of spherical and rodlike micelles. At intermediate $\mathrm{pH}$, the system can be considered a mixture of nonionic monomers, cationic monomers, and effective dimers formed by hydrogen bonding of the first two. As noted, the double-tailed geometry of the last species is best accommodated in regions of lower surface curvature and therefore it tends to migrate into rodlike regimes. Elongated micelles thus consist of cylindrical domains in which $\beta$ tends to approach 0.5 , with endcaps of $\beta>0.5$ or $<0.5$, depending on whether the stoichiometric or bulk value of $\beta$ is larger or smaller than 0.5. Migration of surfactant can be intermicellar as well as intramicellar; consequently the average value of $\beta$ for a given micelle may be larger or smaller than the stoichiometric average. The absence of short rodlike micelles suggests that were such species to exist, they would disproportionate, two short rodlike micelles combining to form a spherical micelle and a longer rod. Spherical micelles best accommodate nonionic or cationic monomers, both of which have relatively large effective headgroups, whereas $\beta=0.5$ dimers tend to collect in cylindrical domains. While nonionic surfactant monomers and fully protonated monomers thus both may stabilize domains of high curvature, and $\beta=0.5$ dimers may stabilize rodlike domains, neither one stabilizes zones of negative curvature where the two domains meet. The driving force for the disproportionation could thus be the elimination of the unfavorable regime of inverse curvature where swollen endcaps meet cylindrical regions of lesser radii. The following section provides a theoretical account of such proposed mechanisms.

\section{Theoretical Section}

Self-Consistent Field Theory: Preliminaries. The state of the art of understanding cylindrical micelles is already rather sophisticated. On the basis of a detailed evaluation of the grand potential of micelles, Eriksson argued convincingly that the end caps of cylindrical micelles must have a larger diameter than their cylindrical body. ${ }^{39}$ Porte et al. ${ }^{40}$ have suggested that there should be a gap in the distribution of wormlike micelles, between the spherical micelles and the long micelles, because of the more unfavorable state of the surfactants that are at the junction between the cylindrical body and the endcaps, i.e., at the neck of the micelle. A gap in the size distribution naturally leads to the concept of the second CMC. The second CMC indicates the rather abrupt appearance of rodlike micelles in equilibrium with (almost) spherical micelles, both, in turn, in equilibrium with free monomers. These coexistences have been reported, for example, by Bernheim-Groswasser et al. ${ }^{32}$ for gemini surfactant solutions. The concept of an endcap with structural properties different from the middle is widely accepted 

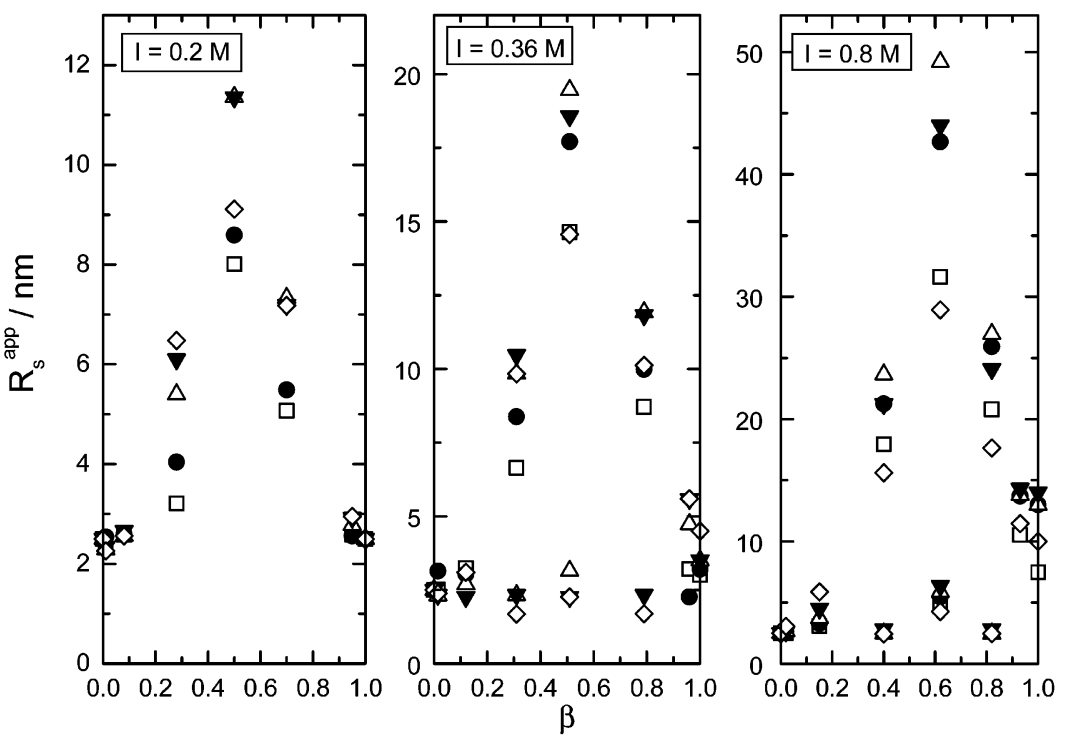

Figure 6. Variation of $R_{\mathrm{s}}{ }^{\text {app }}$ with degree of protonation $(\beta)$ as a function of $I$ and [DMDAO]: $\square, 5 \mathrm{mM} ; \bullet, 10 \mathrm{mM} ; \Delta, 25 \mathrm{mM} ; \boldsymbol{\nabla}, 50 \mathrm{mM} ; \diamond$, $100 \mathrm{mM}$. Coexistence of rods and spheres is observed at $0.3<\beta<0.8$, for $I>0.3 \mathrm{M}$; (Protein Solutions system). For explanations see text.

TABLE 1: Comparison of Apparent Radii (nm) Obtained for $25 \mathrm{mM}$ DMDAO in $0.8 \mathrm{M} \mathrm{NaCl}$, at Different $\mathrm{pH}$, Using Different Deconvolution Algorithms

\begin{tabular}{clll}
\hline $\mathrm{pH}$ & $\mathrm{NNLS}^{a}$ & CONTIN $^{b}$ & REPES $^{c}$ \\
\hline 3 & $10 \pm 0.5$ & $14 \pm 0.7$ & $5 \pm 0.25$, \\
& & & $20 \pm 1.0$ \\
4 & $6 \pm 0.3$, & $2.5 \pm 0.1$, & $5 \pm 0.3$, \\
& $22 \pm 1$ & $27 \pm 1.4$ & $20 \pm 1.0$ \\
5 & $7.5 \pm 0.4$, & $6 \pm 0.3$, & - \\
& $31 \pm 1.6$ & $49 \pm 2.5$ & \\
6 & $4 \pm 0.2$, & $2.5 \pm 0.1$, & $3 \pm 0.2$, \\
7 & $15 \pm 0.8$ & $24 \pm 1$ & $20 \pm 1$ \\
8 & $4 \pm 0.2$ & $3.7 \pm 0.2$ & - \\
& $3 \pm 0.2$ & $2.7 \pm 0.1$ & $4 \pm 0.2$, \\
9 & $4 \pm 0.2$ & - & $19 \pm 1$ \\
& & & $3 \pm 0.2$
\end{tabular}

${ }^{a}$ Brookhaven BIC-9000AT. ${ }^{b}$ Protein Solutions Model 801. ${ }^{c}$ ALV500.

in the literature and the positive endcap (free) energy plays an essential role in explaining the exponential size distribution of wormlike micelles. ${ }^{41}$ One may estimate the value of the endcap energy from the grand potential of the spherical micelles that exist at the same chemical potential for the surfactants as their threadlike counterparts. Below we will see that this guess is indeed rather accurate.

The foregoing comments suggest that the coexistence of spheres and rods in micellar solutions is not limited to DMDAO or gemini systems. Indeed we anticipate that this is a generic phenomenon. If this is true, one should be able to find evidence for such phenomena from molecular modeling. In the following we report self-consistent field calculations, which have the unique property that there is no constraint on the molecules other than their topology. This topology constraint finds its necessity in the need to specify how the local mean-field approximation is implemented. In contrast to the method of Eriksson we do not need to specify the position of the headgroups in any arbitrary way. This allows the system to equilibrate in all aspects related to the distribution of surfactant between cap, neck, and body of the rodlike micelles and the bulk solution. It is possible to evaluate accurately the grand potential in those systems and from this obtain unique information on the stability of wormlike micelles as a function of the aggregation number, leading to a prediction of the endcap free energy as well as a gap in the size distribution. Since the self-consistent field method has been explained in detail, ${ }^{42-47}$ only the main characteristics necessary to understand our predictions will be presented below.

The Discretization Scheme. Solving the self-consistent field equations relies on a discretization scheme; we use that of Scheutjens and Fleer. ${ }^{42,43}$ In short it features a 3-dimensional lattice in which a mean-field approximation is implemented in at least one ${ }^{47,48}$ but in its most simple form in two dimensions. ${ }^{42-44,49}$ Since concentration gradients can be obtained in two or one direction, respectively, and we refer to these as $2 \mathrm{D}$ and 1D SCF models. Molecules are assumed to be composed of spherical units matching the size of the lattice sites. As the symmetry of the lattice is pre-assumed, this choice dictates the shape or topology of the micelles. Three cases are briefly reviewed as follows.

The classical 1D SCF model is first used to consider the spherical ${ }^{45}$ and (infinitely long) cylindrical topologies. ${ }^{47}$ In the latter we will consider all relevant properties per unit length of the cylinder and consider the radial component of the various distributions in the system only. In these lattices there is an origin at $z=0$ and layers with spherical or ring-like topology, respectively, contain $L(z) \sim z$ sites, where $z=0,1,2, \ldots, z_{M}$. For the spherical micelles again the radial distributions are of interest where $L(z) \sim z^{2}$. These two systems will be used to compute the physical properties of the middle part of long cylindrical micelles and spherical ones. Note that in these geometries the shape fluctuations are not accounted for. The 2D SCF analysis involves a cylindrical coordinate system, with the $z$-axis in the direction of the long axis of the cylinder and the $R$-coordinates go in the radial direction. Again the $R=0$ coordinate is the central one, and it suffices to consider only positive values of $R=0,1,2, \ldots, R_{M}$. The numbering of the $z$-coordinate is arbitrary and runs from $z=0, \ldots, z_{M}$. The $R_{M}$ and $z_{M}$ coordinates are the last ones considered in the system. The value is chosen such that at these coordinates homogeneous bulk properties prevail. The number of lattice sites at coordinate $(z, R)$ is given by $L(z, R)$ and is linear in the value of $R$, i.e., $L(z, R) \sim R$ (circle of lattice sites). Mirrorlike boundary conditions are imposed on $z=0$ and $z=z_{M}$, as well as on $R_{M}$. The rodlike micelles are "generated" along the axis of the 
cylinder, where $z=0$ is the midpoint of the rodlike micelle. As conformational fluctuations of the wormlike micelles are ignored, we will refer to the surfactant assemblies as rodlike.

The Molecules. Surfactant molecules are introduced with segments $s=1, \ldots, 16$ being apolar, and segments $s=17, \ldots$, 20 polar; in short $\mathrm{C}_{16} \mathrm{H}_{4}$. Together with the interaction parameters discussed below, the surfactant leads to a surfactant packing parameter ${ }^{51} P \sim 1 / 2$. Because the aim of the theory is to model the generic aspects of the sphere to rod transition in micellar solutions, we do not require at this level replication of DMDAO. We note that one $\mathrm{C}$ unit stands for a united atom $\mathrm{CH}_{2}$ or $\mathrm{CH}_{3}$, which means that the characteristic size of a lattice site must be on the order of $0.3 \mathrm{~nm}$. The solvent $\mathrm{W}$ (water) is a complex medium in itself. Because dealing with the hydrogen-bonding properties of water is computationally expensive, we assume water to be composed of dimers $\mathrm{W}_{2}$. This choice is not critical in the following results, and we refer to the literature for a more detailed motivation for this. ${ }^{52}$

The SCF Approach Tailored to Surfactant Systems. In each coordinate $r$, i.e., value of $z$ in the 1D theory and for each set $(z, R)$ in the $2 \mathrm{D}$ theory, there exists for each segment type $\mathrm{B}$ in the system (here $\mathrm{B}=\mathrm{C}, \mathrm{H}, \mathrm{W}$ ) on one hand a potential field $u_{\mathrm{B}}(r)$ and complementary to this an observable volume fraction $\varphi_{\mathrm{B}}(r)$.

To a good approximation an aqueous solution is incompressible and therefore it is assumed that $\sum_{\mathrm{B}} \varphi_{\mathrm{B}}(r)=1$ for all coordinates $r$. This constraint leads to a Lagrange field contribution $^{44}$ to the segment potential, which is denoted by $u^{\prime}(r)$. The second contribution to the segment potential is due to shortrange nearest-neighbor contact energies parametrized by FloryHuggins interaction parameters. In the present system there are three of these given by $\chi_{\mathrm{CH}}, \chi_{\mathrm{CW}}$, and $\chi_{\mathrm{HW}}$. The first one, which accounts for the repulsion between head and tail segments, is fixed as $\chi_{\mathrm{CH}}=2.2$. The higher its value the less overlap between tail and headgroup profiles will be seen. The second FH parameter accounts for the driving force for micelle formation. The value $\chi_{\mathrm{CW}}=1.4$ is chosen so that the predicted CMC is reasonably close to an experimental one. The final one accounts for the headgroup water contacts. To have a sufficient stopping mechanism for the micellar growth, an attractive interaction is chosen, here chosen as $\chi_{\mathrm{HW}}=-0.7$. Again, the exact values of the parameters are not essential in our arguments, but as will be shown below, are consistent with relative stable cylindrical micelles. Without going into further detail, we mention that the segment potential becomes a function of the segment densities and are normalized to be zero in the bulk solution. ${ }^{44}$

On the other hand, the volume fraction distributions follow (in a unique way) from the segment potentials. To do so, a Markov approximation is applied in which chain connectivity is accounted for by the requirement that segments $s$ and $s-1$ occupy neighboring sites on the lattice. ${ }^{53}$ In this method, chain conformations are not self-avoiding, i.e., it is allowed that $s-$ 1 and $s+1$ occupy the same site. The advantage of the Markov approximation is that the evaluation of the volume fraction profiles become extremely inexpensive as a propagator scheme and can be implemented to find the statistical weight of all possible and allowed conformations. ${ }^{39,44}$

The set of equations is solved by a computer. ${ }^{44}$ The selfconsistent solution has the property that the volume fractions that determine the segment potentials are recomputed by a propagator scheme that uses the segment potentials. The same applies for the potentials: they both follow from and determine the volume fraction profiles. For such a stationary point (selfconsistent solution) it is possible to evaluate the partition

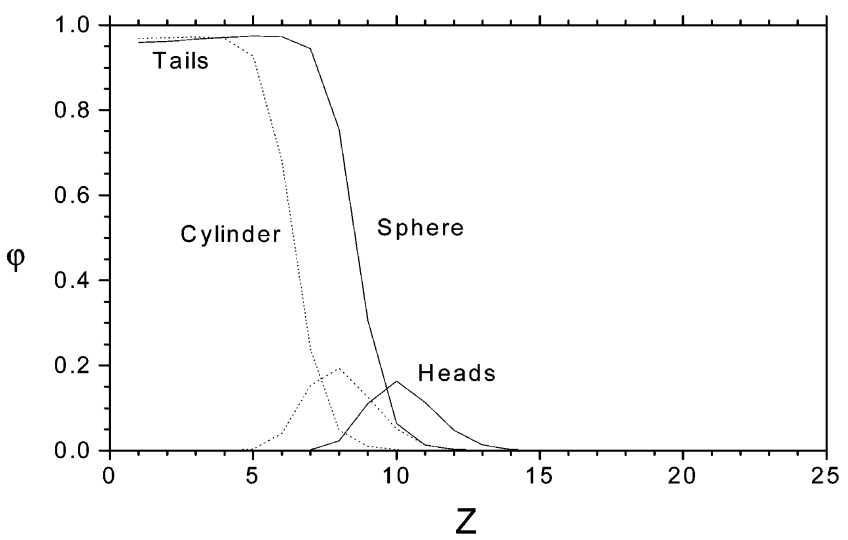

Figure 7. 1D-radial density distribution of tail- and head-group segments of $\mathrm{C}_{16} \mathrm{H}_{4}$ surfactant in spherical and cylindrical geometry. FH interaction parameters are given in the text. The bulk concentration of surfactants is the same in the two cases to $\varphi^{b}=0.00015919$. The grand potential per unit length of the cylindrical micelle is zero, that of the spherical micelle is given by $\epsilon_{m}=5.6157 k T$. The aggregation number (per unit length) in the cylinder geometry is $n^{\sigma} / h=9.947$, and the aggregation number in the spherical micelle is given by $n^{\sigma}=143.1$.

function, and from this, all thermodynamic quantities such as the Helmholtz energy, the grand potential, and the chemical potentials. ${ }^{44}$ Typically, the densities are obtained with 7 significant numbers and the grand potential has at least 5 significant numbers.

\section{Results}

1D results. Let us first analyze the properties of the infinitely long cylindrical micelles. The first point is that, per unit length of the cylindrical micelle, translational entropy is vanishingly small. In addition, within the mean-field approach the conformational entropy of these threadlike micelles is ignored. With this, it follows that the grand potential of the wormlike micelle per unit length is essentially zero. Figure 7 presents the radial density distribution of tails and headgroups of such equilibrated (i.e., tensionless) rodlike micelles. The number of surfactant molecules per unit length is about 10 , and the volume fraction of surfactants in the bulk amounts to $\varphi^{b}=0.000159$. The radial density profile of surfactant micelles is as expected; the tail density is high in the core (small $z$ values) and the headgroups are collected on the outside of the structure. There is little overlap between tails and heads. The water profile (not drawn) may be found by subtracting the tail and head volume fractions from unity.

For spherical micelles translational entropy cannot be ignored. For this reason it follows that the grand potential $\epsilon_{\mathrm{m}}$ of the translationally restricted micelle must be positive. The volume fraction of micelles is given by ${ }^{54,55} \varphi_{\mathrm{m}}=\exp -\epsilon_{\mathrm{m}} / k T$. It can be shown ${ }^{55}$ that micelles are only stable if

$$
\frac{\partial \epsilon_{\mathrm{m}}}{\partial n^{\sigma}}<0
$$

where $n^{\sigma}$ is the excess number of surfactants in the micelle. As there exists a Gibbs-Duhem relation

$$
\frac{\partial \epsilon_{\mathrm{m}}}{\partial \mu}=-n^{\sigma}
$$

where $\mu$ is the chemical potential of the surfactant, it follows that the stability of micelles dictates that 


$$
\frac{\partial \mu}{\partial n^{\sigma}}>0
$$

Equation 9 thus states that when the aggregation number goes up, the chemical potential of the surfactant must increase as well. If eqs 7 and 8 do not apply, one has a local maximum in the free energy and the micelles are instable. In Figure 7 we show the radial density distribution of stable micelles that exist at the bulk volume fraction of surfactant of $\varphi^{b}=0.00015919$. The translationally restricted grand potential of these micelles is found to be $\epsilon_{m}=5.6 \mathrm{kT}$. In the first-order theory we thus expect that long wormlike micelles with two endcaps (half a spherical micelle) will have endcap energies of approximately $2.8 k T$. The radial density distributions of spherical micelles are comparable to that of the cylindrical ones; the cores and coronas have the same characteristics. There exist noteworthy differences between spherical and cylindrical micelles. Interestingly, there is a slight depression in segment density in the core of the spherical micelle, which seems to be less pronounced for the cylindrical geometry. The headgroup density has a slightly larger value for the cylindrical geometry than for the spherical one. The fact that the differences are small indicates that the area per molecule is rather insensitive to the micelle geometry. A more important difference is that the radius of the spherical micelles is significantly larger than that of the cylinder. The maximum of the headgroup profile is shifted by approximately 2 layers (i.e., about $0.6 \mathrm{~nm}$ ). If the endcaps of the rodlike micelles are similar to (half) the spherical micelle and the middle part similar to the cylindrical cross-section, we must indeed expect that the endcap will have a larger radius than the middle part.

2D Results. In Figure 8 we give a typical two-gradient density distribution of a rodlike micelle as obtained by the $2 \mathrm{D}$ theory. For clarity we give the distribution not only for the positive values of $(z, R)$ but also included the mirror images in the $-z$ and $-R$ direction. The results are presented in terms of equal density contour plots as well as gray scale plot, where the high density is given by black and the low density as white. Both the tail and headgroup density profiles are presented. To our knowledge such two-gradient distributions of rodlike micelles have not yet appeared in the literature. As expected, the endcaps have a larger diameter than the middle. Moreover the contour plots give proof of the existence of a neck: going from the endcap to the body of the rodlike micelle, one invariably enters a region with negative Gaussian curvature. This is relatively unfavorable (apparently the Gaussian bending modulus is negative) for the surfactants and this leads to a small depression in the radius. From visual inspection, one can estimate that the length along the long axis of the rod over which the depression is visible is of the order of the size of a spherical micelle.

One cannot know the thermodynamic relevance from a single "snapshot" as given in Figure 8. Indeed this is the problem of MD simulations for which typically just one micelle is generated in the simulation box. ${ }^{55}$ However, the SCF results are computationally inexpensive and it is easy to generate rodlike micelles with arbitrary length, i.e., micelles that differ with respect to their aggregation number.

In Figure 9 we present the results for the translationally restricted grand potential as a function of (half) the aggregation number (i.e., we consider only the part of the rodlike micelle with positive $z$-values). We present the data as obtained by the calculations without any data handling. The result for the grand potential is rather complicated. The first point to notice is that there exists a "high frequency" oscillation. Roughly, with the addition of 10 extra surfactants to the aggregate the grand potential completes a sine wave whose amplitude increases linearly with $n^{\sigma}$. Physically the rodlike micelle with 10 extra surfactants increases in length by one lattice site. With the shift of the endcap with one unit along the $z$-axis, there exists one relatively good (match) and one relatively poor (mismatch) position with respect to the underlying lattice. As a result, the grand potential wiggles. We note that the chemical potential of the surfactant necessarily changes likewise as a cosine function (cf. eq 8); when the grand potential is at a local maximum the chemical potential is at a minimum and the other way around. As these oscillations are due to the lattice, there is no physical significance to it. Apart from these short wavelength oscillations there are features on a longer length scale which are of interest.

From Figure 9 we see that the limiting value of the grand potential (of half the rodlike micelle) goes to approximately $3.25 k T$. This may be interpreted as the endcap free energy, which is very close to the value $2.8 \mathrm{kT}$ predicted from the $1 \mathrm{D}$ results. For these relatively long rods the surfactant concentration in the bulk approaches very accurately the expected limiting value; it oscillates around $\varphi^{b}=0.000159$.

The amplitude of the (long wavelength) oscillations of $\epsilon_{m}$ decreases with increasing length of the worm, indicating that this feature should be linked to the interaction between the two ends of the rodlike micelle. Figure 9 shows regions where $\epsilon_{m}$ is an increasing function of the aggregation number, and regions where it is a decreasing function. Only the decreasing parts represent stable micelles as explained above. Inspection of Figure 9 shows that there are at least three instable regions, while the remaining parts represent (at least a locally) stable micelles. The oscillations in $\epsilon_{m}\left(n^{\sigma}\right)$ resemble the oscillations often seen for solvents confined within two surfaces, wherein these oscillations, which decay exponentially, are rationalized as structural forces. Here the density of lipids is high and the influence of the endcaps on the cylindrical part between the ends also vanishes exponentially in an oscillatory way. The wavelength of the oscillations is about $\Delta n^{\sigma} / 2=35$ or $\Delta n^{\sigma}=$ 70. As the number of surfactants per unit length in the cylindrical micelle is about $10, \Delta n^{\sigma}=70$ corresponds to 7 lattice units, i.e., about $2.1 \mathrm{~nm}$, matching approximately the radius of the spherical micelle (see Figure 7). We will discuss the features of Figure 9 from low to high aggregation number.

-For $n^{\sigma} / 2<120$, the number of surfactants of one-half of the rodlike micelle is significantly less than that of a spherical micelle ( $n^{\sigma}=140$ as given in Figure 7 ). This means that the overall rod has less than twice the aggregation number of the spherical micelle. From the thermodynamic analysis, we learn that these highly deformed dumbbell-like micelles are instable.

-For $120<n^{\sigma} / 2<167$, the deformation of the dumbbell is gradually removed, yielding the first region of thermodynamically stable asymmetric micelles. At the upper limit, the aggregation number of one-half the rodlike micelle exceeds that of the spherical micelle by about 25 surfactants. Increasing the aggregation number further will introduce a cylindrical middle part.

- For $167<n^{\sigma} / 2<205$, the rodlike micelles are unstable. This instability can be ascribed to the existence of a neck between endcap and cylindrical body as disclosed in Figure 8. The overlap of necks on both sides of the rod effectively leads to attraction between the endcaps. The stability is only recovered when the aggregation number of half the rod is about $150 \%$ that of the spherical micelle. This means that the overall length of the rod is about 3 times the diameter of the spherical micelle, i.e., about $12 \mathrm{~nm}$. 

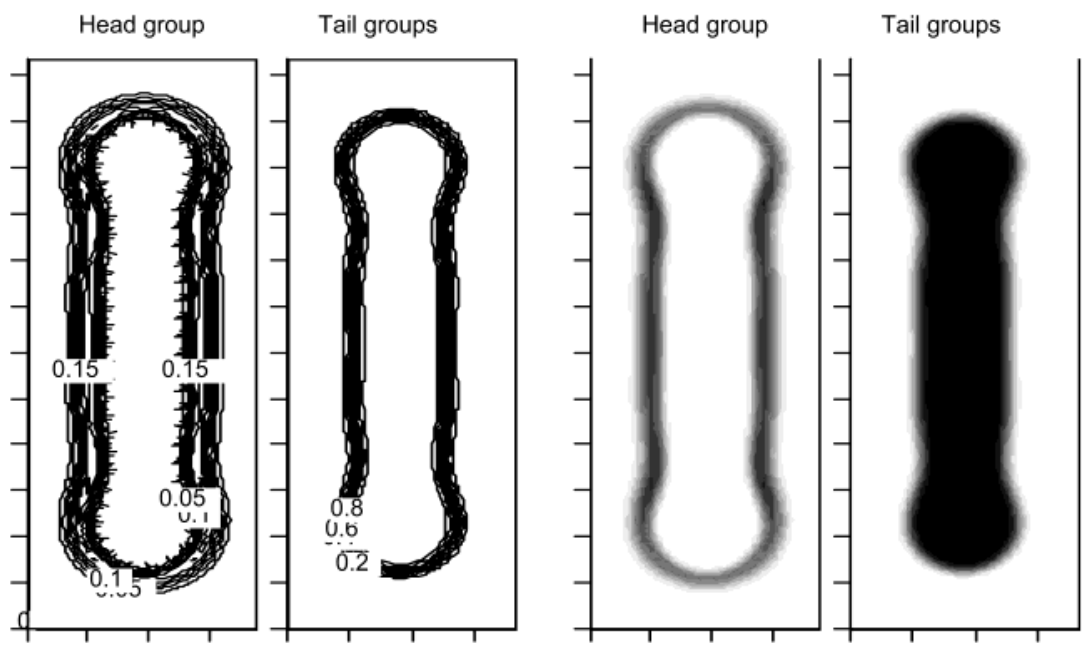

Figure 8. Density distributions as found by the $2 \mathrm{D}$ SCF theory. Left: Both head-group and tail group equal volume fraction contour lines of a cross-section through the center of a linear wormlike micelles with a length/width ratio of about 7 . Right: The density map across the central plane of the wormlike micelle; head-group and tail groups are shown separately. Ramp: high density $=$ black, low density $=$ white. The number of surfactants in this rodlike micelle is given by $n^{\sigma}=550 . z_{M}=50$ and $R_{M}=15$. Also the mirror-images are plotted, i.e., total grid shown $-z_{M}<$ $z<z_{M},-R_{M}<R<R_{M}$

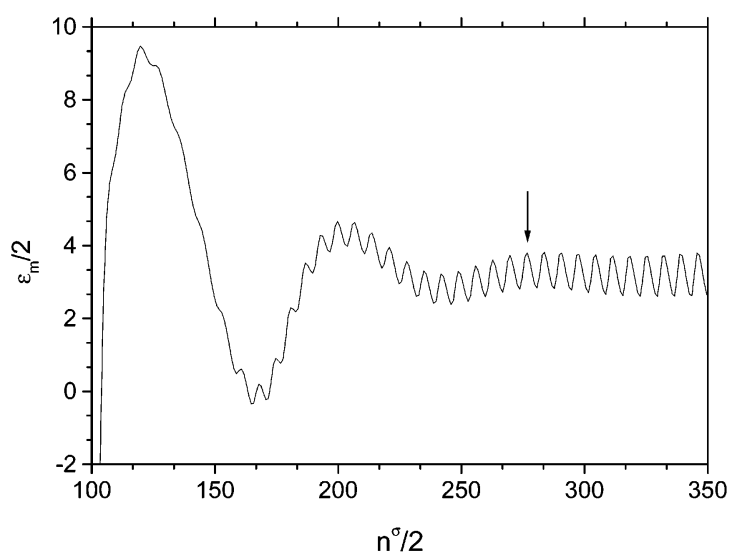

Figure 9. The translational restricted grand potential of half the rodlike micelle $\epsilon_{m} / 2$ as a function of the aggregation number of half the rodlike micelle $n^{\sigma} / 2$. The arrow points to the rodlike micelle presented in Figure 8. For large values of $n^{\sigma}$ the volume fraction of free surfactant in the bulk oscillates around $\varphi^{b}=0.00015919$.

-In the range $205<n^{\sigma} / 2<240$, the overall length of the rodlike micelle increases by 7 lattice units, a size range corresponding to stable micelles. Inspection of the density profiles of the cylindrical part of these micelles (not shown) indicates that the radius of the cylinder in the central part does not converge to a fixed value yet, but is still an increasing function of $n^{\sigma}$.

-Less easy to see is the next instable region $240<n^{\sigma} / 2<$ 275 . For some very subtle reason, the grand potential is here again an increasing function with aggregation number. Apparently in this size range the conformations of the surfactants in the cylindrical part are still perturbed by the proximity of the endcaps (structural perturbations propagate along the cylinder).

-In the remainder, i.e., $n^{\sigma} / 2>275$, any further oscillations are so small that they cannot be detected with reasonable accuracy by our method. Moreover, these long spherocylindrical objects are expected to be in the range of the persistence length, ${ }^{47}$ and the conformational entropy on the level of the wormlike micelle starts to play a role. In effect, this suggests that the micelles with a larger aggregation number than approximately 550 are all stable. The rodlike micelle given in Figure 8 is near the upper limit of the last instability regime (indicated by the arrow in Figure 9).
The fact that the size distribution of rodlike micelles is not continuous gives rise to the idea that the sphere-to-rod transition is first-order-like. The unstable rod lengths discussed present free energy barriers that need to be overcome to go from spherical to rodlike micelles. These free energy barriers also rationalize the coexistence between spheres and rods with finite length. Our prediction, more detailed than experimental observations in the literature, yields at least two gaps in the size distribution.

\section{Conclusions}

The preceding very general treatment shows (1) micelles with aggregation numbers intermediate between those of spheres and those of rodlike micelles form dumbbell-shaped aggregates in which the swollen endcaps are of larger diameter than the cylindrical regions, and (2) that such micelles are energetically unfavorable relative to either spheres or long rods. It may be construed that this result corresponds to unfavorable negative curvature at the interface between endcaps and cylinders.

The model used here is extremely general, containing essentially only parameters characterizing the pair-wise interactions of head-solvent, tail-solvent, and tail-head, along with rather general molecular geometries. Not taken into account, but present in the experimental system are (1) the specific hydrogen bonding between uncharged and charged monomers, leading to the simultaneous presence of at least two of the three species: uncharged monomer, cationic monomer, and the hydrogen-bonded dimer which can behave effectively as a cationic double-tailed surfactant species; and (2) the electrostatic interactions among the last two components. Explicit coexistence between rods and spheres cannot be shown by the SCF method because this would require some mixed geometry. The coexistence between spheres and rods can only be deduced from a thermodynamic analysis of the system. For this reason, it is remarkable that the model agrees so well with experiment in explaining the absence of ca. $10 \mathrm{~nm}$ diameter micelles. The introduction of additional parameters that reflect the presence of several surfactant subunit species is an additional part of a more refined treatment. The fact that these subunits all have particular and different preferences for regions of high and low surface curvature introduces a constraint on the relative amounts of spherical and cylindrical regions in the entire system. If the 
relative concentrations of the three species mentioned above can be determined as a function of ionic strength and degree of protonation, a very precise agreement between theory and experiment may be attained.

Acknowledgment. P.L.D. acknowledges support from NSF DMR 0076068.

\section{References and Notes}

(1) ACS Symposium Series, 501(Mixed Surfactant Systems); Holland, P. M., Rubingh, D. N., Eds.; American Chemical Society: Washington, DC, 1992.

(2) Ikeda, S.; Tsunoda, M.; Maeda, H. J. Colloid Interface Sci. 1979 70,448 .

(3) Zhang, H.; Dubin, P. L.; Kaplan, J. I. Langmuir 1991, 7, 2103.

(4) Herrmann, K. W. J. Phys. Chem. 1962, 66, 295.

(5) Corkill, J. M.; Hermann, K. W. J. Phys. Chem. 1963, 67, 934.

(6) Hermann, K. W. J. Phys. Chem. 1964, 68, 1540.

(7) Benjamin, L. J. Phys. Chem. 1964, 68, 3575.

(8) Tokiwa, F.; Ohki, K. J. Phys. Chem. 1966, 70, 3437. 511.

(9) Goddard, E. D.; Kung, H. C. J. Colloid Interface Sci. 1973, 43,

(10) Maeda, H.; Tsunoda, M.; Ikeda, S. J. Phys. Chem. 1974, 78, 1086.

(11) Funasaki, N. J. Colloid Interface Sci. 1977, 60, 54.

(12) Ikeda, S.; Tsunoda, M.; Maeda, H. J. Colloid Interface Sci. 1978, 67,336 .

(13) Mille, M. J. Colloid Interface Sci. 1981, 81, 169.

(14) Chang, D. L.; Rosano, H. L. In Structure/Performance Relationships in Surfactants; Rosen, M. J., Ed.; ACS Symposium Series 253; American Chemical Society: Washington, DC, 1984; p 129.

(15) Warr, G. G.; Grieser, F.; Evans, D. F. J. Chem. Soc., Faraday Trans. 1 1986, $82,1829$.

(16) Maeda, H. J. Phys. Chem. 1988, 92, 4490

(17) Abe, A.; Imae, T.; Shibuya, A.; Ikeda, S. J. Surf. Sci. Technol. 1988, $4,67$.

(18) Rathman, J. F.; Christian, S. D. Langmuir 1990, 6, 391

(19) Uchiyama, H.; Christian, S. D.; Scameborn, J. F.; Abe, M.; Ogino, K. Langmuir 1991, 7, 95 .

(20) Brackman, J. C.; Engberts, J. B. F. N. Langmuir 1992, 8, 424.

(21) Imae, T.; Hayashi, N. Langmuir 1993, 9, 3385.

(22) Kaimoto, H.; Shoho, K.; Sasaki, S.; Maeda, H. J. Phys. Chem. 1994, 98,10243 .

(23) Maeda, H.; Muroi, S.; Ishii, M.; Kakehashi, R.; Kaimoto, H.; Nakahara, T.; Motomoura, K. J. Colloid Interface Sci. 1995, 175, 497. 263.

(24) Maeda, H. Colloids Surf. A: Physiochem. Eng. Aspects 1996, 109,

(25) Maeda, H.; Muroi, S.; Kakehashi, R. J. Phys. Chem. B 1997, 101, 7378.

(26) Terada, Y.; Maeda, H.; Odagaki, T. J. Phys. Chem. B 1997, 101, 5784.

(27) Garamus, V.; Kameyama, K.; Kakehashi, R.; Maeda, H. Colloid Polym. Sci. 1999, 277, 868 275.

(28) Maeda, H.; Kakehashi, R. Adv. Colloid Interface Sci. 2000, 88 ,

(29) Israelachvilli, J. N. Intermolecular and Surface Forces; Academic Press: New York, 1985; p 256.

(30) Mazer, N. A.; Olofsson, G. J. Phys. Chem. 1982, 86, 4584

(31) Khan, A.; Kaplun, A.; Talmon, Y.; Hellsten, M. J. Colloid Interface Sci. 1996, 181, 191.

(32) Bernheim-Groswasser, A.; Zana, R.; Talmon, Y. J. Phys. Chem. B 2000, 104, 4005 .

(33) Tribet, C.; Porcar, I.; Bonnefont, P. A.; Audebert, R. J. Phys. Chem. B 1998, 102, 1327

(34) Lawson, C. L.; Hanson, R. J. Solving Least Squares Problems; Prentice Hall Inc.: Englewood Cliffs, NJ, 1974. (b) Morison, I. D.; Grabowski, E. F.; Herb, C. A. Langmuir 1985, 1, 496.

(35) Koppel, D. E. J. Chem. Phys. 1972, 57, 4814

(36) Provencher, S. W. Comput. Phys. Commun. 1982, 27, 229.

(37) Jakes. J. Collect. Czech. Chem. Commun. 1995, 60, 1781.

(38) Stepanek, P. Dynamic Light Scattering, The method and some applications; Brown, W., Ed.; Clarendon Press: Oxford, 1993; pp 177240.

(39) Eriksson, J. C.; Ljunggren, S. J. Chem. Soc., Faraday Trans. 2 1985, 81, 1209. (b) Eriksson, J. C.; Ljunggren, S. Langmuir 1990, 6, 895. (40) Porte, G.; Appell, J. J. Phys. Chem. 1981, 85, 2511. (b) Porte, G. J. Phys. Chem. 1983, 87, 3541

(41) Porte, G. Micellar growth, flexibility and polymorphism in dilute solutions. In Micelles, Membranes, Microemulsions and Monolayers, Gelbart, W. M., Ben-Shaul, A., Roux, D., Eds.; Springer-Verlag: New York, 1994.

(42) Scheutjens, J. M. H. M.; Fleer, G. J. J. Phys. Chem. 1979, 83, 1619.

(43) Scheutjens, J. M. H. M.; Fleer, G. J. J. Phys. Chem. 1980, 84, 178

(44) Evers, O. A.; Scheutjens, J. M. H. M.; Fleer, G. J. Macromolecules 1990, $23,5221$.

(45) Leermakers, F. A. M.; Scheutjens, J. M. H. M. J. Colloid Interface Sci. 1990, 136, 231 .

(46) Leermakers, F. A. M.; Rabinovich, A. L.; Balabaev, N. K. Phys

Rev. E: Stat., Nonlinear, Soft Matter Phys. 2003, 67, 011910/1-17.

(47) Lauw, Y.; Leermakers, F. A. M.; Cohen Stuart, M. A. J. Phys Chem. $B$, in press.

(48) Schlangen, L. J. M.; Leermakers, F. A. M.; Koopal, L. K. J. Chem. Soc., Faraday Trans. 1996, 92, 579

(49) Leermakers, F. A. M.; Scheutjens, J. M. H. M.; Lyklema, J. Biochim. Biophys. Acta 1990, 1024, 139

(50) Israelachvilli, J. N.; Mitchell, D. J.; Ninham, B. W. J. Chem. Soc., Faraday Trans. 2 1976, 72, 1525.

(51) de Bruijn, V. G.; van den Broeke, L. J. P.; Leermakers, F. A. M.; Keurentjens, J. T. F. Langmuir 2002, 18, 10467.

(52) Meijer, L. A.; Leermakers, F. A. M.; Lyklema J. J. Chem. Phys. $1999,110,6560$.

(53) Hill, T. L. Thermodynamics of small systems, (Part 1; Part 2); Dover Publications: New York, 1991 (Part 1); 1992 (Part 2).

(54) Hall, D. G.; Pethica, B. A. Nonionic surfactants; Marcel Dekker: New York, 1976; Chapter 16.

(55) Karabonrni, S.; Esselink, K.; Hilbers, P. A. J.; Smit, B.; Karthauser, J.; van Os, N. M.; Zana, R. Science 1994, 266, 254. 